\title{
Interaction with autonomy: Multiple Output models and the inadequacy of the Great Divide
}

\author{
Julie E. Boland ${ }^{\mathrm{a}, *}$, Anne Cutler ${ }^{\mathrm{b} * *}$ \\ '137 Townshead Hall, 1885 Neil Avenue, Ohio State University, Columbus, OH 43210, USA \\ ${ }^{\mathrm{b}}$ Max Planck Institute for Psycholinguistics, Post Box 310, NL-6500 AH Nijmegen, \\ Netherlands
}

Received 14 September 1994, final version accepted 1 July 1995

\begin{abstract}
There are currently a number of psycholinguistic models in which processing at a particular level of representation is characterized by the generation of multiple outputs, with resolution - but not generation - involving the use of information from higher levels of processing. Surprisingly, models with this architecture have been characterized as autonomous within the domain of word recognition but as interactive within the domain of sentence processing. We suggest that the apparent confusion is not, as might be assumed, due to fundamental differences between lexical and syntactic processing. Rather, we believe that the labels in each domain were chosen in order to obtain maximal contrast between a new model and the model or models that were currently dominating the field. The contradiction serves to highlight the inadequacy of a simple autonomy/interaction dichotomy for characterizing the architectures of current processing models.
\end{abstract}

Models of psycholinguistic processing typically consist of a number of levels loosely corresponding to levels of linguistic analysis. Even where a model deals only with the operations of one level-for example, word recognition or parsing - some assumptions about its relationship to the other levels will usually be spelled out. In part, this is because models virtually always take a stand on one side or the other of what has come to form a Great Divide in psycholinguistic theorizing: interaction versus autonomy.

Consider models of syntactic processing. One of the defining issues is

\footnotetext{
* Correspondence to either author. J.E. Boland: e-mail jboland@ling.ohio-state.edu. A. Cutler: e-mail anne@mpi.nl.
} 
whether syntactic choices are made with the benefit of relevant semantic knowledge. For example, both sentence fragments in (1) are syntactically ambiguous between a main clause structure (...the book.) and a reduced relative structure (... by the lawyer was informative.).

(1) (a) The defendant examined ...

(b) The evidence examined ...

However, in (1b) there is an asymmetry: it is much more plausible that evidence is being examined than that evidence is examining something. Thus the main clause structure ought to be blocked for (1b) if semantic or pragmatic information can be used to decide between syntactic alternatives. On the other hand, the main clause structure is simpler, so it might be preferred if only syntactic information could be considered. Widely cited work by Ferreira and Clifton (1986) suggested that syntactic decisions such as these are based solely upon structural simplicity, as proposed by Frazier's (1978, 1987) autonomous model of syntactic processing. According to Frazier's model, the parser always constructs the simplest structure allowed by the phrase structure rules of the grammar. This initial parse uses only the major syntactic category (noun, verb, etc.) of the input, and is later checked against detailed lexical and semantic information.

Recent work, however, suggests that semantic influences can affect syntactic choices (e.g., Altmann, Garnham, \& Dennis, 1992; Britt, 1994; Boland, Tanenhaus, Garnsey, \& Carlson, 1995; Pearlmutter \& MacDonald, 1992; Trueswell, Tanenhaus, \& Garnsey, 1994). For example, Trueswell et al. found that although processing difficulty arose when sentences like (1a) were completed with a reduced relative structure, no such difficulty occurred when sentences like (1b) were completed with a reduced relative structure. They argue that, contrary to Frazier's (1987) claims, detailed lexical information is used to constrain the syntactic alternatives, and semantic information is used to select among them. Similar arguments are put forth in Boland et al., based on work on wh-questions.

As might be expected, proponents of the constraint-based lexicalist approach have adopted a position on the question of interaction versus autonomy. They describe their approach as an interactive system, because multiple constraints, some of them non-syntactic, govern the selection of the initial syntactic structure (e.g., MacDonald, Pearlmutter, \& Seidenberg, 1994; Tanenhaus \& Trueswell, 1994).

The incremental interactive theory first proposed in Crain and Steedman (1985) and further refined in Altmann and Steedman (1988) also maintains that semantic processes are involved in syntactic selection. In this model, syntactic alternatives are constructed in parallel within the constraints of lexical specifications, and a single representation is selected by the semantic system, using principles of referential support, a priori plausibility, etc. Thus there is a bottom-up generation of alternatives, with selection of a single structure left for a later stage of processing. 
As the label given to the model makes plain, Altmann and Steedman (1988) considered their model to be interactive, noting that their results "support the interactive hypothesis" (p. 192). However, they explicitly described it as only weakly interactive. "According to this [weak] version [of the interactive hypothesis], syntax autonomously proposes analyses, while semantics and context merely dispose among the alternatives offered" ( $p$. 205). They contrasted their position with strongly interactive models, which generate only the most plausible structure(s), and with Frazier's (1987) autonomous model, which generates only the simplest structure.

Perhaps the principal feature in which the constraint-based lexicalist approach resembles the incremental interactive model is that in both, syntactic alternatives are evaluated in parallel. That is, in each model the syntactic structure generator explicitly produces multiple outputs. Both contrast in this respect with Frazier's model, in which only a single syntactic structure is considered at a time.

The incremental interactive model clearly separates generation processes from selection processes. This distinction is not unique to the parsing literature, however; it is also a feature of many word recognition models. Among models of visual word recognition, the earliest step in this direction was Becker's (1976) "verification model", in which a rough physical analysis of the input extracts sensory features and compiles a set of candidate words having those features, which are compared one by one against a stored sensory representation of the input. In fact, Becker's model contained two separate generation processes; besides the physically appropriate set of candidate words, a semantically appropriate candidate set (also to be compared with the stored input) was generated by a separate process. The "checking model" put forward by Norris (1986) more closely resembled the syntactic models described above, in that it proposed generation of a single initial candidate set. The set, compiled on the basis of partially analyzed perceptual information, is continually updated as the perceptual analysis is refined. In the meantime, however, selection can begin; the candidates in the set are checked for compatibility with the sentential or other semantic context constructed so far in the recognition process. There is no ordering within the candidate set; word frequency, contextual compatibility and perceptual information can all increment individual candidate words' weightings and thus contribute to determining which candidate word first reaches a specified selection criterion.

Models of spoken-word recognition, too, may split the recognition process into separate stages. Norris' (1994) Shortlist model, as its name suggests, is one such; in this model the initial stage again generates multiple candidates compatible with the input, while in the second stage a process of competition (involving, again, adjustment of weightings for each candidate word) determines which of the shortlisted candidates eventually wins through to recognition. (Note that an initial rough analysis of the entire word, as proposed for visual word recognition, is inappropriate for spoken word recognition. In speech, beginnings of words arrive temporally prior to 
middles and ends, and word length cannot be initially apparent. Nevertheless, as Norris (1994, p. 226) points out, Shortlist's initial stage is directly comparable to the initial stage of the checking model in that in each case a candidate set of words is proposed on the basis of only partial bottom-up information.)

The revised version of the cohort model of Marslen-Wilson (1987, 1993) also proposes an initial stage in which only the perceptual input determines a subset of lexical entries. Selection among this set of activated candidates is then carried out by a later stage, which again operates on perceptual information alone, in parallel with a contextual integration stage. Similarly, the Neighborhood Activation Model (NAM; Luce, Pisoni, \& Goldinger, 1990) involves parallel activation of candidate words, with selection among the activated candidates being performed by a separate decision process. As Marslen-Wilson (1987) points out, the concept of multiple output distinguishes such models from, for example, such direct access models as the logogen model (Morton, 1970) in which only one lexical entry will surmount a recognition threshold and be effectively accessed. Norris (1986) argued that incorporating multiple output makes word recognition models in effect more parsimonious, since post-access selection between multiple options is in any case required to deal with the phenomenon of lexical ambiguity. Several seminal papers in the 1970 s showed that under certain conditions presentation of an ambiguous word will lead to momentary availability of its multiple senses (Conrad, 1974; Swinney, 1979; Tanenhaus, Leiman, \& Seidenberg, 1979), even when the context renders only one sense acceptable; the implication is that selection of the contextually appropriate sense must occur at a post-access stage. If the mechanism for selection between multiple candidates must exist in any case, for recognition when the input cannot unambiguously determine the output of the access process, then architectural economy is best served by exploiting precisely that mechanism in all recognition processes, for unambiguous as well as for ambiguous words.

There is widespread agreement in the word recognition literature of recent years that in Multiple Output approaches in this area the lexical access process is truly autonomous, that is, operates independently of higher-level processes. Becker's (1976) model, to be sure, allowed for semantic context to drive a lexical access process; but it was a process separate from the input-driven generation of word candidates. In all other models containing a single lexical generation process, the actual process of contacting a lexical entry is responsive solely to bottom-up perceptual information, and is not affected in any way by higher-level processing; this is held to constitute autonomy of the lexical access process. Thus the checking model has "a completely bottom-up flow of information" and the "stages are completely autonomous" (Norris, 1986, p. 131); the revised cohort model constitutes "a fully bottom-up model where context plays no role in ... access and selection" (Marslen-Wilson, 1987, p. 71) because "both 
access and certain aspects of selection are autonomous processes, in the sense that they are driven strictly from the bottom-up" (Marslen-Wilson, 1987, p. 98); Shortlist is "a bottom-up autonomous model" (Norris, 1994, p. 231 ) in which all "top-down feedback ... is redundant" (Norris, 1994, p. 191).

The psycholinguistic literature may appear, to a newcomer, to be prey to internal confusion. Both for parsing and for word recognition, Multiple Output models have been proposed which have basically the same architecture, but models in the two domains take fundamentally incompatible positions when they label themselves with regard to the interaction/autonomy dichotomy. Researchers in each domain clearly agree on the criteria by which these labels are applied, and thus, within each area, there is no confusion; but we believe that the reasons for the asymmetry provide an interesting object of scrutiny.

The label that best fits the Multiple Output architecture depends on what one considers to be the defining features of autonomy and interaction, respectively. In fact, different definitions have been established in word recognition and parsing. In the parsing literature, use of higher-level information to resolve lower-level decisions constitutes interaction, so Multiple Output models are considered interactive because higher-level information is used in the selection process. In word recognition, in contrast, Multiple Output models are considered clearly autonomous because a process is not taken to be interactive unless higher-level information actually affects the way that alternatives are generated within the system, ruling out certain candidates irrespective of their compatibility with bottom-up information. Autonomy would imply that processing operations at a given level proceed in the same way irrespective of whatever counsel might be deducible from higher-level considerations. This type of autonomy, which has characterized the debate within the domain of word recognition, is also the definition that Fodor (1983) used in his argument for modularity in mental processing: "a system [is] autonomous by being encapsulated, by not having access to facts that other systems know about" (p. 73). Whether or not it is given the label "autonomous", this architecture is common to all Multiple Output models. In fact, despite having labeled their model weakly interactive, Altmann and Steedman (1988) point out that the architecture of their parsing model "does not compromise the modularity hypothesis of Fodor (1983) in any way" (p. 192).

The fact that parsing models and word recognition models have maintained different definitions of autonomy provides only a superficial explanation for the inconsistent labeling of Multiple Output models. The question then becomes: why has the parsing literature used one definition and the word recognition literature another? One possible reason is that there exist fundamental differences between lexical and syntactic processing, which justify adopting different definitions of autonomy. Traditionally, word recognition has been viewed as a lookup process, that is, the access of stored 
lexical representations. Parsing, on the other hand, has been viewed as a construction process, whereby representations are computed rather than being chosen from a store. Correspondingly, outputs of lexical processing have been assumed to coincide with the completion of the processing stage (i.e., recognition of the word), but outputs of syntactic processing have been taken to correspond to many incremental stages in the construction of a complete syntactic structure.

However, we believe that current models of both parsing and word recognition make the maintenance of such rigid distinctions no longer tenable. For instance, it is clear that processes which essentially involve simple lookup can do much of the work in parsing traditionally believed to require construction processes. There is abundant evidence that syntactic decisions make use of detailed lexical information that is accessed as part of word recognition. This research has focused primarily on verb-based information, such as subcategorization frames (e.g., McElree, 1993; Osterhout, Holcomb, \& Swinney, 1994), verb control information (Boland, Tanenhaus, \& Garnsey, 1990), and thematic roles (e.g. Britt, 1994; Mauner, Tanenhaus, \& Carlson, 1995; Stowe, 1989; Taraban \& McClelland, 1988). Use of stored lexical information means that syntactic processing is more dependent upon access processes and less dependent upon construction processes than has often been assumed. MacDonald et al. (1994) have taken the lexicalist approach to sentence processing even further, suggesting that the lexical entries of nouns, verbs, and words of other categories contain $\mathrm{X}$-bar structures. The only construction that takes place in their model is the connecting of one X-bar structure to another.

On the other hand, models of word recognition-and, in particular, Multiple Output models-do not necessarily consist solely of lookup procedures. For instance, Norris" "checking model" (1986) of visual word recognition contains much more of a continuous element, in that the initial stage is continually outputting updated analyses to the checking stage. Likewise, the Shortlist model of spoken word recognition (Norris, 1994) provides for a continuous input from the initial generation stage to the competition/selection stage. In fact this continuous updating feature turns out to be an essential feature of Shortlist. In order to account for empirical data indicating that human listeners employ prelexical segmentation routines in conjunction with competition processes (McQueen, Norris, \& Cutler, 1994), the Shortlist model has been modified to include a prelexical segmentation procedure mimicking Cutler and Norris' (1988) Metrical Segmentation Strategy (Norris, McQueen, \& Cutler, 1995). To achieve this, it proved essential that the updated output of the initial stage continually replace the previous output; only with this replacement mechanism did the model succeed in simulating the human empirical data. The continuous output feature of such models renders the notion of a simple lookup procedure, with its completion amounting to completion of the lexical stage of processing, inaccurate as a description of the word recognition process. 
We do not mean to imply that there is agreement that lexical and syntactic processes are fundamentally alike; these issues remain the subject of hot debate, and the traditionally held differences may in fact have influenced the adoption of different definitions of autonomy in the two domains. But whatever the outcome of the debate, there is no longer any logical force behind the argument that lexical and syntactic processing are so different that an identical architecture motivates opposite theoretical descriptions in the two domains.

Instead, we believe that considerations outside the architecture of processing models have influenced how Multiple Output models have come to be labeled. Multiple Output models, both of word recognition and of parsing, were introduced after other models had already, in effect, defined the territory. In each case, the Multiple Output model posed a challenge to the existing model, and was correspondingly assigned an opposing label.

The dominant model in syntactic processing in the 1980s, when the syntactic models discussed above were first mooted, was undoubtedly Frazier's (1978) model. Moreover, Frazier's model was particularly known for its position in the dominant theoretical debate in psycholinguistics, in that it was declared to be strictly autonomous. Thus the opposing models which were indeed very different in structure - came to be termed interactive; this emphasized that Multiple Output models formed a genuine theoretical alternative to the currently dominant approach. In fact, as we shall argue below, it is possible to claim that the proposed alternative models actually embodied more autonomy than Frazier's model, in which the autonomy is strictly limited in scope; nevertheless, the label "interactive" provided the most effective contrast.

In the word recognition literature, too, we believe that labels were influenced by considerations of contrast. Just as autonomy could be said to be making the running in syntactic modeling, and hence be the position with which contrast could most easily be drawn, so were there models in word recognition which were influential in much the same way, and these models were interactive. In visual word recognition, the dominant model prior to the emergence of Multiple Output models was Morton's (1970) logogen model, in which higher-level information from the context contributed directly to the activation of lexical candidates just as bottom-up information from incoming input did. In spoken-word recognition, the logogen model was also a contender, but the first model specifically devoted to the auditory case, the original Cohort model of Marslen-Wilson and Welsh (1978), was likewise perceived to be fully interactive, with syntactic and semantic context capable of controlling the initial availability of potential candidate words. (In fact, a careful exegesis of the 1978 model can interpret it as containing the seeds of its later revision in Multiple Output form. But the model was not fully specified; it was termed "interactive access"; and no distinction was drawn between access and selection. Claims such as "... top-down/bottom-up interactions are not ... a re-working of a first pass 
over the input, but are instead the primary operations that produce the ... percept"' (p. 44) led readers to place the model firmly on the interactive side of the Great Divide.) Finally, TRACE (McClelland \& Elman, 1986), the most influential model of spoken-word recognition since the mid-1980s, again embodies interactive use of higher-level information in lower-level processing during the word recognition process. Thus emphasizing autonomy in the architecture again allowed proponents of Multiple Output models to achieve maximal contrast with certain currently dominant models.

The interaction/autonomy debate functioned as a rather effective energizer for psycholinguistics in the last few decades; it may have stimulated more research than any other single issue. Placing one's contribution within this paradigm has been de rigueur; but, as we have argued, the placement may not always have been rigorously determined by architectural issues alone. Contrast with theoretical alternatives - that is, in effect, sociopolitical considerations - may have played as large a role. The result of these joint pressures to take a stand on one or other side of the Great Divide and to achieve maximum contrast with alternatives has been an apparently contradictory labeling of basically identical architectures as autonomous within one framework and as interactive within another.

One simple conclusion to be drawn from this state of affairs, we would argue, is that the Great Divide no longer provides an adequate classificatory system for psycholinguistic models. The essential differences among model architectures in the mid-1990s cannot be captured by a simple distinction between interaction and autonomy. Finer distinctions are necessary, with architectural claims specifying whether processing is interactive or autonomous at each stage of processing (for instance: generation of outputs, selection between candidate outputs, recovery from a mis-selection), and whether or not there are multiple (parallel) outputs at each stage.

In spoken-word recognition, Shortlist, NAM and the revised Cohort model are autonomous in the generation of initial lexical candidates; candidates are activated solely on the basis of information from the signal. TRACE, on the other hand, is interactive in that the presence of top-down connections allows the structure of the lexicon itself to exercise an influence on the input from the phoneme level which flows up to the lexicon. (Were TRACE integrated into a full model of sentence comprehension, inter-level connections could in the same fashion allow even the initial generation of lexical candidates to be directly constrained by semantic and syntactic context). In the selection process, all four models allow some interaction (the Cohort model perhaps least, TRACE most, with Shortlist and NAM falling in between). Revision of the process consequent upon mis-selection does not play an explicit role in word recognition models, but again only TRACE's architecture allows for interactive adjustment within an ongoing generation process; the other models have no options for revision but to re-run the selection process with increased higher-level input (and if applicable with an expanded but still autonomously generated candidate set). 
We have defined Multiple Output models as those in which a single processing stage passes more than one alternative on to a later stage where further decisions are made. Shortlist, the current Cohort model, and NAM, as we have argued, all clearly allow Multiple Output. In TRACE, on the other hand, there is no separation between stages such that any one stage decides upon outputs from an earlier stage; all stages are connected such that processing at any stage has automatic consequences for processing at stages both above and below. This is a principal feature of TRACE, and McClelland and Elman specifically reject any idea of a decision procedure external to the integrated perceptual process (1986, p. 74). Certainly, TRACE allows for activation of more than one lexical candidate at once, and the process of selection between candidates is achieved in exactly the same way in TRACE and in Shortlist, by a process of direct competition between candidates. Thus multiple activation is true of all four of these current models of spoken-word recognition. Multiple Output, in the sense that a prior stage completes its work by passing on more than one alternative for a later stage to select between, is true of only three.

In parsing, Frazier's (1987) model is autonomous in terms of generation and selection of a syntactic analysis during the first pass, with no multiple outputs. However the restricted nature of the initial analysis predicts frequent "garden paths", or parsing errors, and the necessary recovery process is clearly not autonomous. When the initial analysis is inconsistent with thematic information, syntactic reanalysis occurs within, or is guided by, a thematic processor (Rayner, Carlson, \& Frazier, 1983; Ferreira \& Henderson, 1991). Note that it is not enough for the thematic processor simply to send an error signal to restart the syntactic processor, because the syntactic processor would automatically construct the simplest structure once again. Multiple Output models, such as the incremental interactive model (Altmann \& Steedman, 1988), do not have this limitation. If necessary, the syntactic processor would reproduce the parallel outputs exactly as it had the first time, and the external selection processes would make the correct selection, guided by the knowledge of the previous mistake. Thus the Multiple Output parser generates structures completely autonomously during reanalysis as well as during initial analysis, but selection amongst structures is interactive.

The constraint-based lexicalist models are architecturally similar to incremental interactive models, but, like TRACE, have not made clear distinctions between generation and selection. Instead, they emphasize that much structural information can be encoded lexically, so that the output from the lexicon makes syntactic alternatives available to higher-level processes. These alternatives are believed to be weighted by probabilistic information (such as the relative frequency of subcategorization frames for a particular verb); other constraints, such as consistency with semantic and discourse information, also influence which alternative wins out. It is not clear, at this juncture, whether semantic and pragmatic constraints actually limit the alternatives that are considered, thus constituting interaction 
during the generation of candidates. MacDonald et al. (1994, p. 697) suggested that "most contexts probably are weakly constraining, in the sense that they provide an effective basis for deciding between a small number of alternatives but are less effective in isolating a single alternative in advance". However, the selection process is clearly held to be interactive. For example, Spivey-Knowlton and Sedivy (1995) argue for interactive selection on the basis of the finding that referential context can affect how verb preferences determine prepositional phrase attachments.

Thus it is not clear that any current model of parsing is interactive in terms of generation. The differences among parsing models are principally to be found in whether or not they allow multiple outputs, and in how selection among alternatives is performed. In word recognition, in direct contrast, the possibility of multiple output is not a feature that differentiates models, and nor is the autonomy of the selection stage, but the autonomy of the initial generation stage is a distinguishing feature (further important differences arise in the mechanisms by which selection is performed; see McQueen, Cutler, Briscoe, \& Norris, 1995, for detailed discussion).

It is clear that current models cannot be adequately categorized with holistic labels; distinctions between them may involve several dimensions. By drawing our attention to the increasing subtlety of inter-model differences, the apparent contradiction in how Multiple Output models have been defined in psycholinguistics can therefore perhaps prompt a very beneficial outcome for the field: it may help us to abolish the once useful, but now too simplistic, Great Autonomy/Interaction Divide.

\section{Acknowledgements}

This paper was written while J.E. Boland was a Visiting Fellow at the Max Planck Institute for Psycholinguistics. We thank Dennis Norris, Michael Tanenhaus, Lee Osterhout, and two anonymous reviewers for their insights and comments on an earlier draft of this manuscript. The ordering of the authors is alphabetical.

\section{References}

Altmann, G.T.M., Garnham, A., \& Dennis, Y. (1992). Avoiding the garden path: eye movements in context. Journal of Memory and Language, 31, 685-712.

Altmann, G.T.M., \& Steedman, M. (1988). Interaction with context during human sentence processing. Cognition, 30, 191-238.

Becker, C.A. (1976). Allocation of attention during visual word recognition. Journal of Experimental Psychology: Human Perception and Performance, 2, 556-566.

Boland, J.E., Tanenhaus, M.K., \& Garnsey, S.M. (1990). Evidence for the immediate use of verb control information in sentence processing. Journal of Memory and Language, 29, 413-432. 
Boland, J.E., Tanenhaus, M.K., Garnsey, S.M., \& Carlson, G.N. (1995). Verb argument structure in parsing and interpretation: evidence from wh-questions. Journal of Memory and Language, 34 (in press).

Britt, M.A. (1994). The interaction of referential ambiguity and argument structure: the parsing of prepositional phrases. Journal of Memory and Language, 33, 251-283.

Conrad, C. (1974). Context effects in sentence comprehension: a study of the subjective lexicon. Memory and Cognition, 2, 130-138.

Crain, S., \& Steedman, M. (1985). On not being led up the garden path: the use of context by the psychological syntax processor. In D. Dowty, L. Kartunnen, \& A. Zwicky (Eds.), Natural language parsing (pp. 320-358). Cambridge: Cambridge University Press.

Cutler, A., \& Norris, D. (1988). The role of strong syllables in segmentation for lexical access. Journal of Experimental Psychology: Human Perception and Performance, 14, 113-121.

Ferreira, F., \& Clifton, C., Jr. (1986). The independence of syntactic processing. Journal of Memory and Language, 25, 348-368.

Ferreira, F., \& Henderson, J.M. (1991). Recovery from misanalyses of garden-path sentences. Journal of Memory and Language, 30, 725-745.

Fodor, J.A. (1983). The modularity of mind. Cambridge, MA: MIT Press.

Frazier, L. (1978). On comprehending sentences: Syntactic parsing strategies. Ph.D. thesis, University of Connecticut. Distributed by the Indiana University Linguistic Club.

Frazier, L. (1987). Theories of syntactic processing. In J.L. Garfield (Ed.), Modularity in knowledge representation and natural language processing (pp. 291-307). Cambridge, MA: MIT Press.

Luce, P.A., Pisoni, D.B., \& Goldinger, S.D. (1990). Similarity neighborhoods of spoken words. In G.T.M. Altmann (Ed.), Cognitive models of speech processing: Psycholinguistic and computational perspectives (pp. 122-147). Cambridge, MA: MIT Press.

MacDonald, M.C., Pearlmutter, N.J., \& Seidenberg, M.S. (1994). The lexical nature of syntactic ambiguity resolution. Psychological Review, 101, 676-703.

Marslen-Wilson, W.D. (1987). Functional parallelism in spoken word recognition. Cognition, 25, 71-102.

Marslen-Wilson, W.D. (1993). Issues of process and presentation in lexical access. In G.T.M Altmann \& R. Shillcock (Eds.), Cognitive models of speech processing: The second Sperlonga meeting (pp. 187-210). Hillsdale, NJ: Erlbaum.

Marslen-Wilson, W.D., \& Welsh, A. (1978). Processing interactions and lexical access during word-recognition in continuous speech. Cognitive Psychology, 10, 29-63.

Mauner, G., Tanenhaus, M.K., \& Carlson, G.N. (1995). Implicit arguments in sentence processing. Journal of Memory and Language, 34, 357-382.

McClelland, J.L., \& Elman, J.L. (1986). The TRACE model of speech perception. Cognitive Psychology, 18, 1-86.

McElree, B. (1993). The locus of lexical preference effects in sentence comprehension: a time-course analysis. Journal of Memory and Language, 32, 536-571.

McQueen, J.M., Cutler, A., Briscoe, T., \& Norris, D.G. (1995). Models of continuous speech recognition and the contents of the vocabulary. Language and Cognitive Processes, 10, 309-331.

McQueen, J.M., Norris, D.G., \& Cutler, A. (1994). Competition in spoken word recognition: spotting words in other words. Journal of Experimental Psychology: Learning, Memory and Cognition, 20, 621-638.

Morton, J. (1970). A functional model for memory, In D.A. Norman (Ed.), Models for human memory (pp. 203-254). New York: Academic Press.

Norris, D.G. (1986). Word recognition: context effects without priming. Cognition, 22, 93-136.

Norris, D.G. (1994). Shortlist: a connectionist model of continuous speech recognition. Cognition, 52, 189-234.

Norris, D.G., McQueen, J.M., and Cutler, A. (1995). Competition and segmentation in spoken word recognition. Journal of Experimental Psychology: Learning, Memory and Cognition, 21, 1209-1228. 
Osterhout, L., Holcomb, P.J., \& Swinney, D.A. (1994). Brain potentials elicited by gardenpath sentences: evidence of the application of verb information during parsing. Journal of Experimental Psychology: Learning, Memory and Cognition, 20, 786-803.

Pearlmutter, N., \& MacDonald, M.C. (1992). Plausibility effects in syntactic ambiguity resolution. Proceedings of the 14th annual meeting of the Cognitive Science Society (pp. 498-503). Hillsdale, NJ: Erlbaum.

Rayner, K., Carlson, M., \& Frazier, L. (1983). The interaction of syntax and semantics during sentence processing: eye movements in the analysis of semantically biased sentences. Journal of Verbal Learning and Verbal Behavior, 22, 358-374.

Spivey-Knowlton, M.J., \& Sedivy, J.C. (1995). Resolving attachment ambiguities with multiple constraints. Cognition, 55, 227-267.

Stowe, L. (1989). Thematic structures and sentence comprehension. In G.N. Carison \& M.K. Tanenhaus (Eds.), Linguistic structure in language processing (pp. 319-357). Dordrecht: Kluwer.

Swinney, D.A. (1979). Lexical access during sentence comprehension: (re)consideration of context effects. Journal of Verbal Learning and Verbal Behavior, 18, 645-660.

Tanenhaus, M.K., Leiman, J., \& Seidenberg, M. (1979). Evidence for multiple stages in the processing of ambiguous words in syntactic contexts. Journal of Verbal Learning and Verbal Behavior, 18, 426-441.

Tanenhaus, M.K., \& Trueswell, J. (1994). Sentence comprehension. In J. Miller and P. Eimas (Eds.), Handbook of perception and cognition: Speech, language and cognition (pp. 217262). San Diego: Academic Press.

Taraban, R., \& McClelland, J.L. (1988). Constituent attachment and thematic role assignment in sentence processing. Journal of Memory and Language, 27, 597-632.

Trueswell, J.C., Tanenhaus, M.K., \& Garnsey, S.M. (1994). Semantic influences on parsing: use of thematic role information in syntactic ambiguity resolution. Journal of Memory and Language, 33, 285-318. 